\title{
Preventing traffic accidents with in-vehicle decision support systems - The impact of accident hotspot warnings on driver behaviour
}

\author{
Benjamin Ryder ${ }^{\mathrm{a}, *}$, Bernhard Gahr ${ }^{\mathrm{b}}$, Philipp Egolf ${ }^{\mathrm{a}}$, Andre Dahlinger ${ }^{\mathrm{b}}$, Felix Wortmann ${ }^{\mathrm{b}}$ \\ a Information Management, ETH Zurich, Weinbergstrasse 58, Zurich 8092, Switzerland \\ b ITEM-HSG, University of St. Gallen, Dufourstrasse 40a, St. Gallen 9000, Switzerland
}

\section{A R T I C L E I N F O}

\section{Article history:}

Received 20 September 2016

Received in revised form 20 April 2017

Accepted 4 May 2017

Available online 6 May 2017

\section{Keywords:}

In-vehicle decision support systems

Location analytics

Accident hotspots

\begin{abstract}
A B S T R A C T
Despite continuous investment in road and vehicle safety, as well as improvements in technology standards, the total amount of road traffic accidents has been increasing over the last decades. Consequently, identifying ways of effectively reducing the frequency and severity of traffic accidents is of utmost importance. In light of the depicted challenge, latest studies provide promising evidence that in-vehicle decision support systems (DSSs) can have significant positive effects on driving behaviour and collision avoidance. Going beyond existing research, we developed a comprehensive in-vehicle DSS, which provides accident hotspot warnings to drivers based on location analytics applied to a national historical accident dataset, composed of over 266,000 accidents. As such, we depict the design and field evaluation of an in-vehicle DSS, bridging the gap between real world location analytics and in-vehicle warnings. The system was tested in a country-wide field test of 57 professional drivers, with over $170,000 \mathrm{~km}$ driven during a four-week period, where vehicle data were gathered via a connected car prototype system. Ultimately, we demonstrate that in-vehicle warnings of accident hotspots have a significant improvement on driver behaviour over time. In addition, we provide first evidence that an individual's personality plays a key role in the effectiveness of in-vehicle DSSs. However, in contrast to existing lab experiments with very promising results, we were unable to find an immediate effect on driver behaviour. Hence, we see a strong need for further field experiments with high resolution car data to confirm that in-vehicle DSSs can deliver in diverse field situations.
\end{abstract}

(C) 2017 Elsevier B.V. All rights reserved.

\section{Introduction}

According to the World Health Organization (WHO) road traffic accidents are the eighth leading cause of death globally. Moreover, since 2001 the number of road traffic fatalities steadily increased to over 1.2 million people each year [65]. An example of the growing risk can be seen in the USA, where, according to the National Highway Traffic Safety Administration (NHTSA), the number of deaths from traffic accidents in 2015 rose by $7 \%$ from the year before, up to 35,092 [36]. Aside from the humanitarian concerns of so many injuries and fatalities, the worldwide economic costs caused by the impact of traffic accidents are estimated to account for a loss of approximately $3 \%$ of the global GDP [28]. As such, the Department of Transport issued a call to action, encouraging the continuous research into different approaches that can help to reduce the number of traffic accidents, both fatal and non-fatal.

\footnotetext{
* Corresponding author.

E-mail addresses: bryder@ethz.ch (B. Ryder), bernhard.gahr@unisg.ch (B. Gahr), egolfp@student.ethz.ch (P. Egolf), andre.dahlinger@unisg.ch (A. Dahlinger), felix.wortmann@unisg.ch (F. Wortmann).
}

In light of the depicted challenges, a huge variety of decision support systems (DSSs) have emerged to help tackle these problems. Due to the data requirements and the complexity of urban planning and transportation problems, there has been a growing interest in the use of DSSs to analyse the strategic planning $[8,60]$, the multi-vehicle tactical [48] and the individual vehicle operational levels $[25,44]$. In particular, spatial DSSs have been shown to play a vital role in this domain, enabling a variety of analytics on road infrastructure challenges. As an example, road accident hotspots were evaluated in India, where inadequate development of transport networks led to traffic congestions and accidents. Geo-information technology was used to help examine the location and distribution of hotspots, highlighting the influence of spatial and temporal factors in their formation [41]. In addition, various research studies are geared towards how in-vehicle DSSs can encourage drivers to adapt their driving behaviour when necessary. Latest studies provide promising evidence that these systems can indeed have significant positive effects on driving behaviour and collision avoidance $[23,57,63]$. These systems can be delivered to vehicles through existing mobile or standalone satellite navigation systems [68]. 
While the potential of in-vehicle systems is undisputed, the vast majority of studies have focused on simulation experiments $[33,49]$ and controlled field studies [45,68], typically providing warnings to drivers to prevent a collision with an upcoming vehicle or pedestrian. In this field, the benefit of real world location analytics on traffic accident hotspots as a source for in-vehicle warnings has widely not been addressed. As such, the paper at hand depicts the design and field evaluation of an in-vehicle DSS. In contrast to other in-vehicle studies, location analytics were applied to a national historical accident dataset, composed of over 266,000 accidents, and a complete in-vehicle DSS was developed providing warning interventions to drivers. This system was tested outside of the simulation environment in a country-wide field test of 57 professional drivers, over a period of four weeks, with a total of $170,000 \mathrm{~km}$ driven. In order to assess the impact of the system on driver behaviour and safety, real-time sensor data from the vehicles were collected at a rate of up to $30 \mathrm{~Hz}$ through a connected car prototype system. Ultimately, we demonstrate that in-vehicle warnings of historically dangerous locations have a significant improvement on driver behaviour over time while crossing these hotspots. We additionally see evidence that an individual's personality plays a key role in the effectiveness of in-vehicle DSSs, highlighting the importance of personality when researching DSS-based interventions. However, we were not able to confirm an immediate effect of warnings. In essence, we see a strong need for further field experiments with high resolution car data. While there are many lab experiments with impressive results, field evidence is still weak and it remains unsure if in-vehicle DSSs can deliver in diverse field situations on their "lab promises".

The contribution of the paper can be summarised as follows:

1. The in-vehicle DSS that we developed is, to the best of our knowledge, the first of its kind, i.e. incorporating automatically generated accident hotspot warnings as an alternative to human selected locations or up-coming collision warnings.

2. The system is one of the first in-vehicle warning systems to be tested in real world conditions across a longitudinal field study, providing evidence of an improvement on driver behaviour over time.

3. Due to the integration of commonly collected accident location details, the proposed DSS can be easily extended to other parts of the world where such data are compiled, either at a regional or national level.

In the remainder of this paper we outline the related work with regard to in-vehicle DSSs providing warnings to drivers, followed by accident hotspot identification and classification techniques. We then describe the system, with a focus on the location analytics used to identify accident hotspots from historical data, and the generation of the contextual warnings for drivers. Finally, we conclude with an evaluation of the system with regard to its effect on safe driving behaviour, and a discussion of the results and implications of the research.

\section{Related work}

\subsection{In-vehicle warning systems}

The promise of in-vehicle warning systems to improve driving safety has generated a substantial body of research $[2,18]$, and a positive impact is seen when compared with traditional warning approaches in relation to driver behaviour and accident frequency, e.g. in the context of railway crossings [56]. Common conventional warning devices, such as the passive stop sign were compared to active variations, i.e. flashing lights and a half bloom-barrier with flashing lights. The results showed that, on average, driver responses to passive warnings were poor in comparison to active warnings [56]. In a later follow-up study, rumble strips and in-vehicle audio warnings were compared to the previous active and passive warnings at railway level crossings [57]. Results indicated that both the warning devices produced much higher levels of driver compliance than the existing conventional warnings, demonstrating the positive impact in-vehicle warning systems can have when compared to conventional approaches.

Various simulator based studies have shown that in-vehicle warning systems can have a positive impact on driving behaviour. In one example, early warning signals displayed while approaching an intersection showed a positive effect towards driving safer [63]. Participants adapted their driving behaviour by turning with a lower velocity after waiting longer at the intersection, and so avoided collisions. Visual warnings have also had a positive effect on drivers braking reaction time, for both older and younger participants [23]. The largest improvement was seen in critical situations, where collisions were successfully avoided due to the warning. Additionally, there was still a reduction in braking reaction time in less critical situations, where the number of collisions was not a suitable measure of improvement. In other studies, effects on driving behaviour from advisory warnings were found to be strongly dependent on warning time, with earlier warnings more effective than late warnings [33]. Warnings were much more greatly appreciated by drivers when given earlier, even though in critical situations shorter warning times were still effective [34]. In situations where there is low visibility of potential hazardous situations, the frequency of critical situations was reduced when early advisory warnings were provided, especially in surprising or unexpected situations [32]. With regard to the types of warning that can be provided, contextual warnings had limited importance to the behaviour of the driver, but users rated the system much higher due to them [33]. Additionally, it has been shown that in less critical situations a contextual caution warning sign is more suitable than a stop sign warning [23].

Outside of simulation studies, a few controlled field studies have investigated the impact of in-vehicle warnings on driver safety. The influence of warning expectancy and automation complacency on real-life emergency braking has been investigated [45]. In particular, reliable warnings quickened the decision making process and misleading warnings generated automation complacency, slowing visual search for hazard detection. Additionally, specific spatially located hazards have been investigated with regard to the effect of in-vehicle DSSs [68]. The hazardous area tested was an intersection near an arch-shaped bridge, where traffic accidents had often occurred due to poor visibility. The effects of different combinations of audio and visual warnings provided to a driver was investigated. It was demonstrated that information about the cause of accidents was more effective than information on road infrastructure in helping drivers to avoid dangerous driving situations.

\subsection{Accident hotspot analysis}

Over the past sixty years, the topic of road accident hotspot analysis has been extensively researched and various hotspot identification (HSID) methods have been developed. Historically, the most common approaches have been non-spatial techniques, capturing details on the underlying road structure and considering traffic accidents which occurred on these defined sections of road. Probably the simplest identification technique of this type is the so-called Crash Frequency (CF) method [9]. In this approach, a road segment's perilousness is determined by the number of accidents which occurred on it during a specified period. An example of this approach examined the frequency of highway accidents, on the basis of a multivariate analysis of roadway geometries (e.g. horizontal and vertical alignments), weather, and other seasonal effects [50].

Similar to the concept of Crash Frequency, the Crash Rate (CR) method [17] takes the number of accidents which happened on a road segment into account, but additionally considers the traffic 
volume in its analysis. However, there are some evident drawbacks of both methods, such as not considering random fluctuations of the number of accidents [67]. From a statistical perspective, both CR and CF suffer worse performance when compared to other HSID methods [31,67]. Despite this, both CF and CR are still commonly in use today, with their popularity stemming from the ease of implementation and interpretation. Over time, researchers have developed and utilised statistical models in the analysis of accident hotspots $[30,38]$. Probably the most prominent and applied accident hotspot identification technique using a statistical model is the Empirical Bayesian (EB) method $[15,16]$. Based on statistical evidence it is argued that the EB method outperforms other hotspot identification techniques, including CR and CF [31].

However, classic hotspot identification techniques mostly neglect spatial aspects and patterns of accidents, i.e. the actual locations of individual accidents, regardless of the underlying road structure $[13,64]$.

With the increasing appearance of Geographic Information Systems (GIS) and the larger availability of precise, geo-coded data, as well as digital maps, researchers have started to use spatial data analysis methods for identifying accident hotspots [3,13,37]. This follows the theory that the concentration of individual accidents at certain locations is called forth by a set of common causes - implying a spatial dependence of the accidents $[3,13]$. Underlying causes for such a concentration include weather effects, infrastructure or traffic conditions $[14,31,66]$. The most common spatial accident hotspot identification approaches used are either the K-means clustering technique, spatial autocorrelation or the Kernel Density Estimation (KDE) method. Especially the KDE method has been extensively researched $[11,42,66]$, and in general it is argued that it outperforms other HSID methods, such as the spatial autocorrelation, CF or CR, and might perform equally well as the EB $[13,67]$. In an example using this approach, hotspots on highways were explored and determined with two different methods of KDE analysis and repeatability analysis [11], additionally a GIS was used as a management system for accident analysis and determination of hotspots in Turkey with statistical analysis methods. Furthermore, a KDE and a K-means clustering approach were used to profile road accident hotspots [3]. More recently, researchers have started to use the data mining clustering technique Density-Based Spatial Clustering of Application with Noise (DBSCAN), to identify road accident hotspots [53,54]. DBSCAN is a density-based algorithm which classifies elements into clusters in such way that inside a cluster, the density of elements is higher compared to the outside of the cluster [12]. Therefore, it can efficiently identify members of arbitrarily shaped clusters as well as outliers [26].

\section{System description}

We developed an in-vehicle DSS for drivers, that provides contextual warnings on up-coming historically hazardous locations. The architecture of providing these in-vehicle warnings was achieved through the following steps: Firstly, accident hotspots were spatially identified from raw historic accident data. Secondly, these accidents hotspots were then classified into various categories based on the accidents which they were composed of. Finally, the locations and categorisations of the hotspots were provided visually with an in-vehicle DSS in the form of warnings. Fig. 1 depicts this overall process. Additionally, a separate application was developed to review the results of the identification and classification of the accident hotspots. This accident hotspot 'Explorer Tool' was used to validate the parameters of the algorithms chosen, and provides statistical overviews of the accident hotspots, potentially useful for road infrastructure specialists and other street authority decision makers. The remainder of this section outlines the accident hotspot spatial identification and classification techniques employed, and the generation of the in-vehicle warnings.

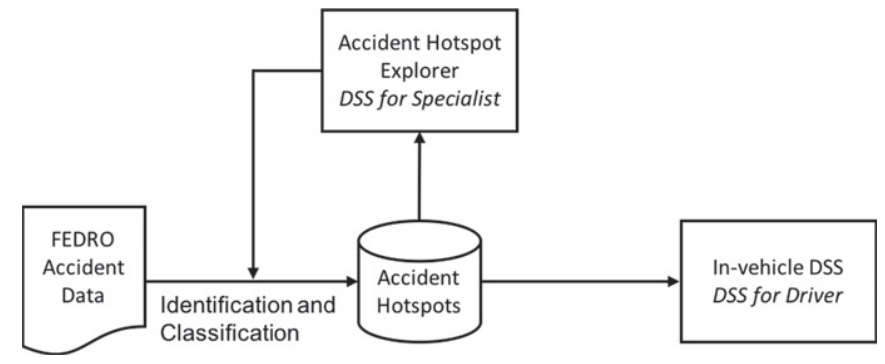

Fig. 1. System overview of the DSS.

\subsection{Accident hotspot identification}

The historical accident dataset was provided by the Swiss Federal Road Office (FEDRO), Statistics For Road Accidents. Since 2011, FEDRO has collected detailed information about every Swiss road accident for which the police were called, building up an extensive accident register. This dataset is composed of over 266,000 geolocated accident records, which occurred in Switzerland between the years 2011-2015. It includes a multitude of features related to each accident, such as the reason for the accident occurring and the surrounding road infrastructure. Many of these features were used to generate appropriate warning feedback for the detected accident hotspots, as will be described in Section 3.2. However, the hotspot identification algorithm required only information regarding the coordinates of the accidents.

The literature review revealed multiple approaches to identify accident hot-spots, however, not all of them were compatible with the available dataset.

The EB approach, which is a commonly applied by governmental institutions, is well known to produce good results. But, it is very sensitive to the quality of the estimation function and requires detailed information about risk variables, such as, traffic volumes or road parameters, e.g. curve radii [10]. Since the provided dataset of the FEDRO consisted only of rough traffic flow estimates and lacked other road parameters, EB was discarded as a suitable approach. Promising spatial analysis tools such as spatial autocorrelation or Kernel Density Estimation are appropriate for identifying locations where many accidents happened. However, current research suggests that spatial clustering techniques can achieve similar results, while being simpler and perform much more efficiently on large datasets [54]. As it is not trivial to identify the hotspot boundaries that KDE generates, and therefore identify the accidents which contribute to a hotspot, DBSCAN was selected as a natural density based clustering technique which clearly identifies observations contributing to a cluster.

DBSCAN classifies elements into clusters in such a way that inside a cluster the density of elements is higher compared to the outside of the cluster [12]. Elements, which are not part of any group, are considered as noise. As such, identified clusters can be considered hotspots with a significantly higher density of accidents compared to other areas. Noise elements represent "random" accidents, which have no, or very little, spatial dependencies to other crashes. DBSCAN's performance in identifying clusters is very sensitive to the distance between points considered part of the same cluster $(\varepsilon)$, and the minimum number of points which must be within $\varepsilon$ to form together a cluster (MinPts). There exists no optimal choice of these parameters, and domain expertise is suggested to identify optimum values based on the intentions of the analysis. If $\varepsilon$ is too small, only accidents occurring in very close proximity to each other will be considered as hotspots, and if too large, hotspots can grow in size and cover parallel roads. Likewise, if MinPts is too high, only the most severe clusters are identified, and if too low, many small and "random" hotspots are found. Therefore, the following practical approaches were considered when applying DBSCAN on the FEDRO 
dataset. The value of MinPts was discussed with experts from one of the largest automotive clubs in Europe and hence defined with the following heuristic: To call a specific location a hotspot, on average, more than two accidents per year had to occur at that location. As a result, it was decided that MinPts cannot be smaller than ten as the hotspots were formed out of a dataset covering accidents over five years. Finally, $\varepsilon$ was fine-tuned by a visual inspection of a selection of accident hotspots through the previously mentioned 'Explorer Tool'. Some of these accident hotspots were closely connected to certain road infrastructure, and others were more spatially distributed. It was found that with $\varepsilon=15 \mathrm{~m}$ DBSCAN produced results where individual clusters did not span multiple roads. These parameters can be loosely defined by the following natural definition: For an observation to be included in an accident hotspot, at least ten accidents must have occurred within $15 \mathrm{~m}$ of that location over five years. With these parameter settings for $\varepsilon$ and MinPts, a total of 1608 unique accidents hotspots were found in Switzerland from over 266,000 geo-located accident records.

\subsection{Accident hotspot classification}

The in-vehicle DSS should provide drivers with warning feedback whenever they are approaching an accident hotspot. Following the guidelines from the NHTSA, drivers should be provided with warning messages in the form of signs and non-critical supporting text [7]. The feedback information varies depending on the available contextual information derived from the spatially identified accident hotspots. The assumption is that drivers can directly and quickly relate the warning sign to the upcoming dangerous location. Additionally, the warning text should provide further non-time critical information, e.g. the predominant cause of the accident hotspot.

The contextual information of each accident hotspot was derived based on the corresponding accident protocols of the FEDRO. In these reports, police officers recorded all related accident information and determined, besides other details, the leading cause and type of the accident. In order to not overwhelm the drivers with too detailed or complicated warnings, a simple categorisation algorithm was developed. The detailed contextual information of each accident was summarised into three main categories: "What", "Why", and "Where". "What" refers to the type of objects which were involved in the accident, e.g. cars, cyclists or pedestrians. "Why" refers to the predominant cause and type of the accident, e.g. disregarding right of way, speeding or swerving. Lastly, "Where" refers to the location information about the predominant type of road infrastructure at which the accident happened, e.g. at a crossroad intersection, roundabout or traffic light. In other words, where possible, information was captured about what objects were involved in the accident, why it happened and where it occurred. Out of this information a warning was generated, with the purpose to provide more information about a specific typical appearance of a hotspot instead of a general warning [23]. It has been shown that warnings making use of contextual objects and directions are preferred by users [33]. Therefore, when generating the warning, the preference of information primarily shown was ranked in the following order: "What", "Why " and "Where".

As such, the warning intervention was generated through a ranked majority-voting of the categorisation statistics of each accident hotspot [27]. In order to capture true contextual information of a hotspot, more than $50 \%$ of the accidents involved had to share the same predominant contextual detail information. Otherwise, a general warning sign and message was shown. Algorithm 1 outlines the classical DBSCAN pseudocode [12], which was modified so that once a hotspot was identified, it was assigned a contextual warning type through the pseudocode provided in Algorithm 2. In the majority of cases the official road warning signs of Switzerland were matched to the generated warnings and were used in the intervention. This was to prevent a potential confusion about the meaning of the shown warning messages. However, in some cases the creation of new symbols was inevitable. In total, six new signs were generated, following NHTSA standards [7]. This classification approach of the 1608 detected hotspots led to a total number of 20 different warnings signs, and 36 unique combinations of sign and text. Fig. 2 shows a selection of four different types of accident hotspot detected using DBSCAN and this classification approach. For brevity the full list of sign and text combinations is omitted, however, the top ten most commonly encountered combinations can be seen in Table 2 , and the full list can be found online [47].

\section{Algorithm 1.}

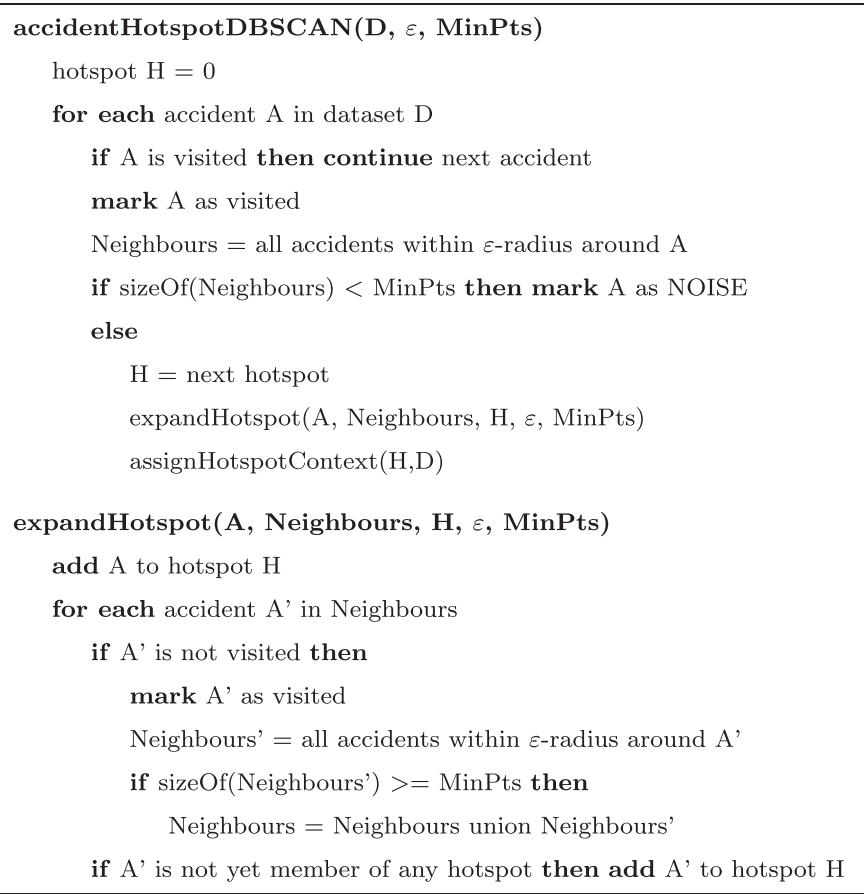

\section{Algorithm 2.}

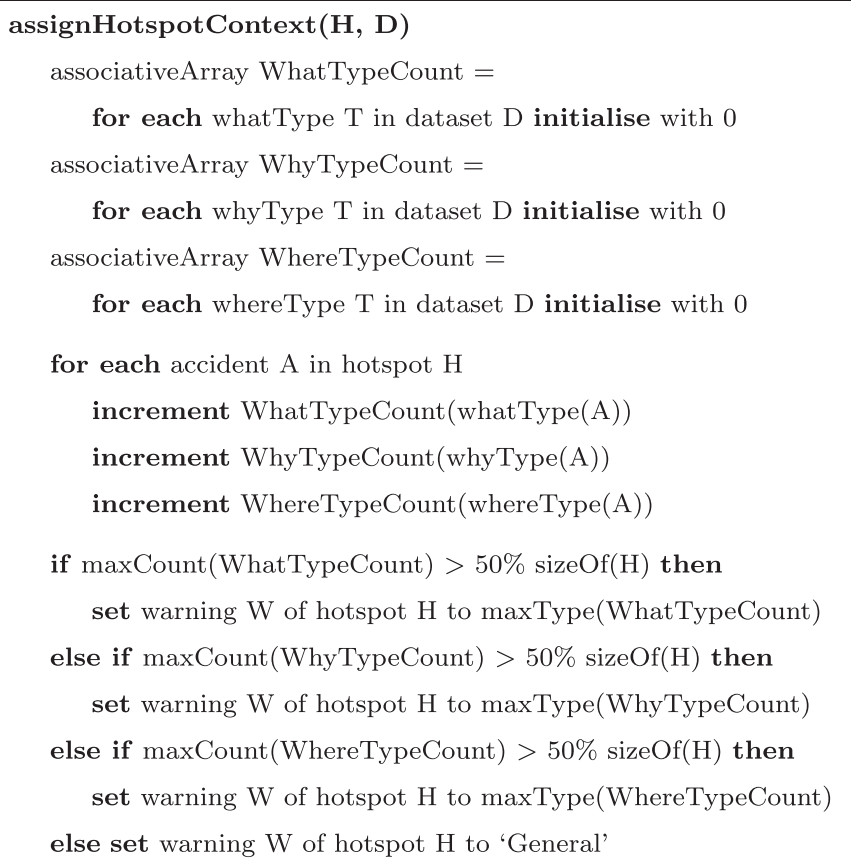




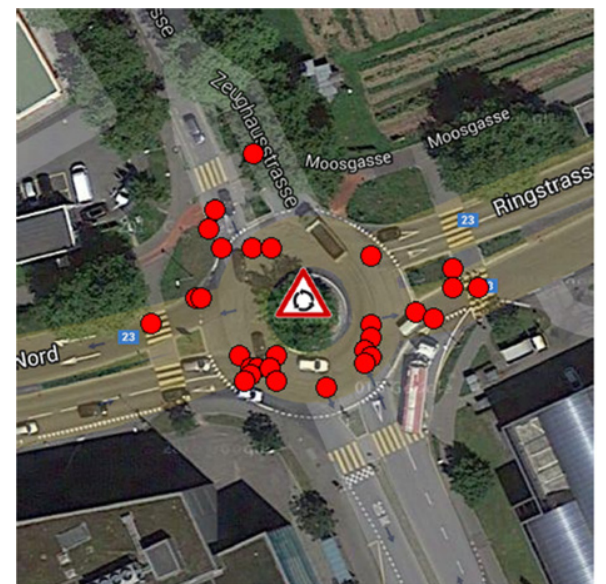

(a) Roundabout Hotspot

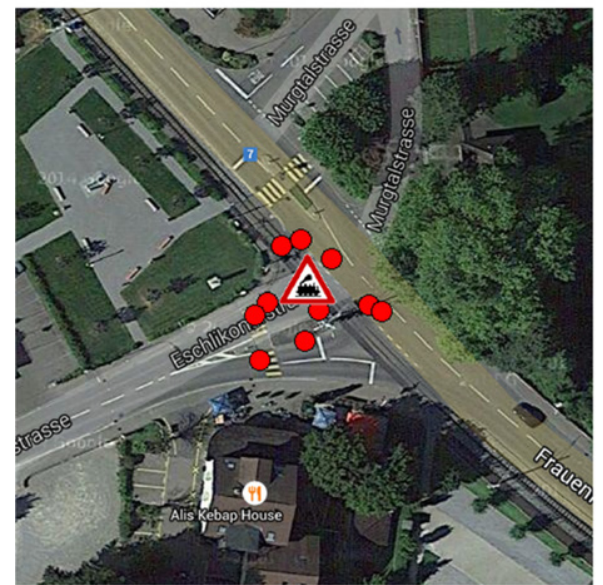

(c) Train Hotspot

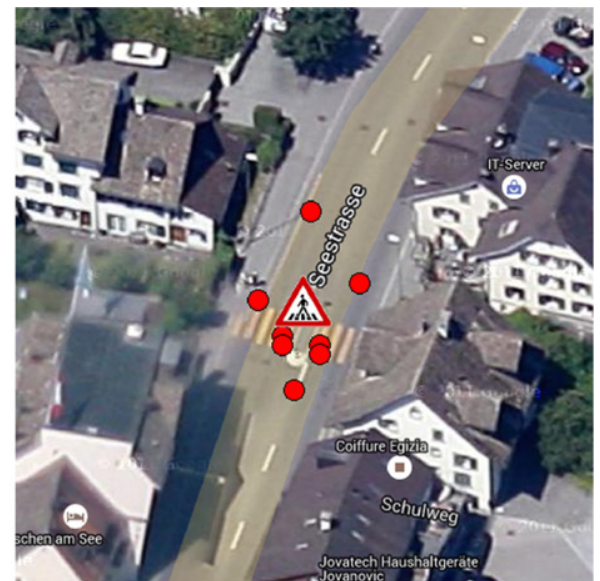

(b) Pedestrian Crossing Hotspot

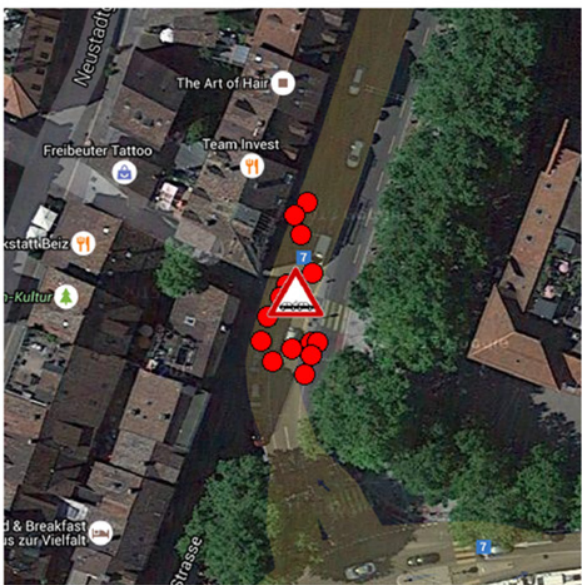

(d) Rear-end Hotspot

Fig. 2. Selection of accident hotspots identified through DBSCAN, accidents contributing to the hotspot are shown in red.

\subsection{In-vehicle warning intervention}

The in-vehicle warning DSS was developed as a native Android application, compatible with Android version 5.0 and later, and builds upon the work of an earlier system [46]. It has been shown that in-vehicle audio-based warnings may be as effective as both audio and visual warning information combined [68]. Additionally, tactile warnings, e.g. vibrations delivered through the vehicle seat, pedal or seat belt, have previously been provided to drivers [29]. However, it was not possible to deliver tactile feedback in our study setting, and it would have been difficult to control for whether the driver had disabled the audio of the smartphone. Thus, only visual

Table 1

Distribution of Big Five personality traits among participants.

\begin{tabular}{llllll}
\hline Personality trait & Mean & $\begin{array}{l}\text { Standard } \\
\text { deviation }\end{array}$ & Median & Minimum & Maximum \\
\hline Agreeableness & 2.85 & 0.65 & 3.00 & 1.00 & 4.50 \\
Conscientiousness & 3.51 & 0.70 & 3.50 & 2.00 & 5.00 \\
Extraversion & 2.91 & 0.76 & 3.00 & 1.50 & 4.50 \\
Neuroticism & 3.50 & 0.65 & 3.50 & 2.00 & 5.00 \\
Openness & 2.94 & 0.56 & 3.00 & 1.50 & 4.50 \\
\hline
\end{tabular}

warnings were implemented as part the DSS in the research at hand.

Regarding visual warnings, the NHTSA released a guideline for the design of crash warning devices [7]. The project reflects a review of the human factors associated with the implementation of such warning system interfaces. The lessons learned were then developed into guidelines for interface design. The highest efficiency was achieved by the choice of a discrete display, providing binary on-off information, and symbol or icon based information. Additionally, the alphanumeric display type lead to poor results and is commented with "Only appropriate for non-time-critical complex information". Based on these design suggestions, the warning sign is primarily displayed with the additional non-time-critical warning text below. Visual warnings are displayed on the smartphone DSS as the driver approaches an accident hotspot, and remain until the area surrounding the hotspot is passed. As earlier warnings are more effective and greater appreciated by drivers than late warnings, the warnings were shown up to $15 \mathrm{~s}$ before a driver encountered an accident hotspot [33,34]. During times that the warnings were not shown, the system displayed eco-driving feedback to encourage use of the DSS. Fig. 3 shows examples of the in-vehicle warning intervention provided to the drivers when approaching an accident hotspot. 
Table 2

Hotspots encountered, and occurrences of one or more heavy braking events, between control and intervention group, for top ten most commonly encountered warnings.

\begin{tabular}{|c|c|c|c|c|c|c|c|}
\hline \multicolumn{2}{|c|}{ Hotspot warning } & \multicolumn{2}{|c|}{ Total } & \multicolumn{2}{|c|}{ Control } & \multicolumn{2}{|c|}{ Intervention } \\
\hline Sign & Text & Count & Events & Count & Events & Count & Events \\
\hline & Disregarding Right of Way & 4541 & 777 & 2135 & 384 & 2406 & 393 \\
\hline & Dangerous Crossroad & 4378 & 783 & 2022 & 354 & 2356 & 429 \\
\hline & Rear-end Collisions & 3532 & 375 & 1407 & 138 & 2125 & 237 \\
\hline & Disregarding Traffic Light & 2037 & 284 & 951 & 120 & 1086 & 164 \\
\hline & Control Speed & 1927 & 87 & 586 & 36 & 1341 & 51 \\
\hline & Caution Dangerous Area & 1471 & 112 & 803 & 60 & 668 & 52 \\
\hline & Swerving Accidents & 964 & 108 & 317 & 35 & 647 & 73 \\
\hline & Disregarding Right of Way & 931 & 200 & 438 & 101 & 493 & 99 \\
\hline & Dangerous Roundabout & 880 & 201 & 407 & 95 & 473 & 106 \\
\hline & Caution Cyclists & 689 & 112 & 342 & 59 & 347 & 53 \\
\hline Other & & 3069 & 349 & 1275 & 150 & 1794 & 199 \\
\hline Total & & 24,419 & 3388 & 10,683 & 1532 & 13,736 & 1856 \\
\hline
\end{tabular}

\section{System evaluation}

\subsection{Participants}

The impact of the in-vehicle warning system on users decision making while driving was assessed through a four week field study of professional drivers, travelling for approximately $4 \mathrm{~h}$ per day in Switzerland. Each of the drivers worked for the same company across a variety of locations, and all drove Chevrolet Captivas of similar make and model. During the four week period over 170,000 km were driven using the system, with an average of $144 \mathrm{~km}$ travelled per driver per day. Of the 72 recruited participants, 57 actively drove during the field study and provided demographic details, such as age and gender. Drivers were randomly allocated to either the control group $(\mathrm{N}=27)$ or intervention group $(\mathrm{N}=30)$. Of these 57 participants, all were male and ranged from 21 to 64 years of age. Using the same categories as previous accident analysis studies [39,40], the majority of drivers (45.61\%) fall between the ages of 35-59 years, with a mean of 40.3 and a median of 39 years of age.

Since prior research indicates that a driver's personality may impact driving behaviour [55], the personality of the participants was measured in order to control for these factors when assessing the effect of the DSS. Therefore, the Big-Five-Inventory-10 (BFI-10) questionnaire [43], a short version of the well-established BigFive-Inventory (BFI) [20], was provided to the drivers. The BFI-10 consists of 10 items to cover the five personality factors, Agreeableness, Consciousness, Extraversion, Neuroticism and Openness, each with two items accordingly and measured on a Likert scale from 1 (very low) to 5 (very high). Psychometric properties do not reach the quality of the original BFI, but deliver sufficient values. The short version of the questionnaire was chosen due to restrictions on participant's time and to avoid attrition. Table 1 provides an overview of the distribution of each of the Big Five personality traits among the drivers. Existing research into the impact of driver personality on driving behaviour shows that various Big Five traits can be linked to four identified driving styles [55]. The results found correlations between 'reckless' and 'angry' driving styles and high levels of Extraversion, and low Agreeableness and Conscientiousness. Additionally, high levels for Agreeableness and Conscientiousness and Openness were correlated to the 'careful' driving style. Finally, the 'anxious' driving style was linked to high Neuroticism.

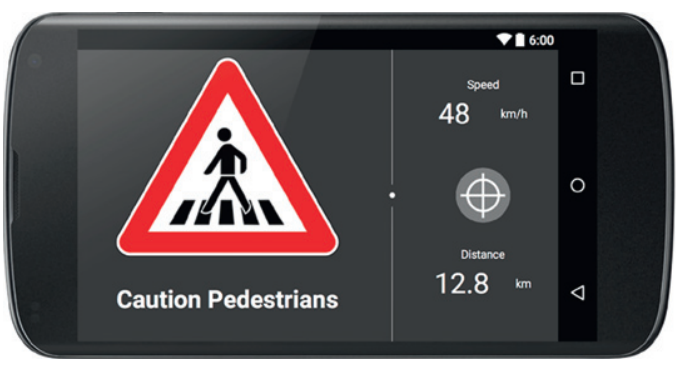

(a) Pedestrian Crosswalk Warning

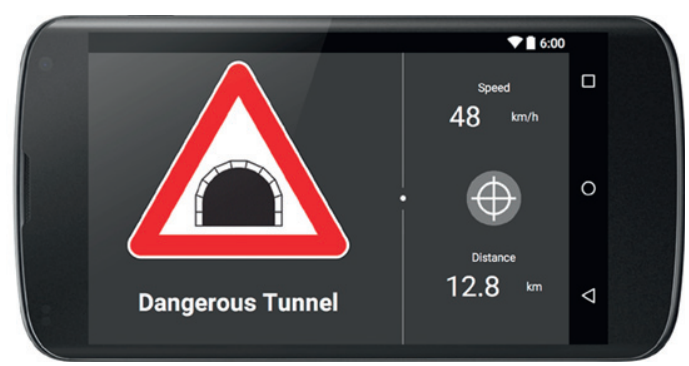

(b) Dangerous Tunnel Warning

Fig. 3. Two examples of the in-vehicle warning intervention shown to drivers approaching an accident hotspot 


\subsection{Field study description}

Existing studies which measure the impact of in-vehicle warnings through simulation environments and controlled field studies made use of a variety of features to assess the effect of warnings on driver behaviour. These include variables such as 'minimum-timeto-collision', the time left for a participant to avoid a collision with another object [35]. However, as the system at hand is tested in the field and does not warn of a specific upcoming object, but instead a historically hazardous location, many of these variables are unavailable to us. For example, measuring number of collisions or braking reaction time [24] is unsuitable, as the distance to other objects is unknown and there may be no need for a change in the driver's behaviour. As such, our dependent variable is the effect of the warnings on potentially dangerous braking events incurred by the driver, which is a key result of decision making driving behaviour. As the DSS encourages awareness of historically dangerous areas, drivers that receive the intervention should be more alert and able to plan ahead more effectively [68]. Therefore, we hypothesise a drop in dangerous braking events.

There are several methods of detecting heavy braking which have been explored in recent literature. A selection of studies have explored the insights that can be gained through smartphone accelerometer data [21]. Unfortunately, this option comes with difficulties, such as drivers interacting with the phone during the journey, triggering high acceleration values and thus leading to false positive events. Therefore, various studies have made use of data which can be extracted from the vehicle itself to measure driving activities. On-board diagnostics (OBD-II) standardised data have been widely used in research as it is mandatory for all vehicles manufactured or sold in the USA from 1996. This data gives insights into features such as vehicle speed, and has been used to detect hazardous driving behaviour [19]. However, specific unstandardised data are available on the Controller Area Network (CAN) Bus of a variety of vehicles, and gives deeper insights into a vehicle's operation. For example, characteristics of aggressive and calm driving have been identified with access to CAN Bus data [22].

As such, we access the CAN Bus of the Chevrolet Captivas involved in the study via an OBD-II dongle. This dongle is paired via Bluetooth with the smartphone in the vehicle. The smartphone then transmits the CAN Bus signals in real-time to a server. For the purpose of this analysis we capture the braking behaviour from the vehicle's longitudinal acceleration. The vehicle speed is additionally collected, calculated from averaging each of the individual wheel speeds. Dangerous braking events have previously been categorised as Low Danger, Dangerous and High Danger levels, based on thresholds of deceleration values [4]. Low Danger events are those where vehicle deceleration was between $1.0-2.0 \mathrm{~m} / \mathrm{s}^{2}$, Dangerous events between $2.0-4.0 \mathrm{~m} / \mathrm{s}^{2}$ and High Danger events as greater than $4.0 \mathrm{~m} / \mathrm{s}^{2}$. As the system provides warnings in historically hazardous locations, we expect a large portion of events to fall into the Low Danger category. Therefore, we consider the deceleration events over $2.0 \mathrm{~m} / \mathrm{s}^{2}$, capturing Dangerous and High Danger level braking events. Only in $0.28 \%$ of the cases where a driver crossed an accident hotspot, more than one dangerous braking event occurred. Thus, a binary measure was applied to generate the dependant variable, i.e. whether or not one or more dangerous events were experienced while the hotspot was encountered.

The evaluation was conducted through collecting vehicle sensor data during times that the warning intervention was shown to the driver. In the case of the control group, data were collected while the warning would have been shown, i.e. when drivers crossed an identified hotspot but no warning was shown. Along with the sensor data, various other variables were collected which have been shown to have an effect on the likelihood of a traffic accident occurring $[39,40]$. These values include the time of day, the day of the week and the speed that the vehicle was travelling when the hotspot was encountered. For comparison, these variables are categorised into bands on the basis of previous studies [39,40]. As shown in Fig. 4, we see that the majority of hotspots were encountered travelling between 30$60 \mathrm{~km} / \mathrm{h}$, with similar distributions between the control and intervention group. Additionally, an incremental count was collected for each driver every time they encountered each specific accident hotspot.

Finally, erroneous observations of encountered hotspots were cleansed from the dataset in certain situations, i.e. where there were issues with the sensors in the vehicle and data were not collected. This led to a total of 24,419 observations of encountered hotspots; 10,683 in the control group where no intervention was provided, and 13,736 in the intervention group where the location based accident hotspot warnings were shown. Table 2 shows the top ten most commonly encountered warning interventions across the study. Additionally, the number of hotspots encountered are shown, as well as occurrences of one or more heavy braking events, for both the control and intervention groups.

\subsection{Analysis and results}

We apply multilevel mixed-effects logistic regression [51] to account for the impact of the individual drivers among the control and intervention groups. Our dependent variable was a binary measure of whether one or more dangerous braking events occurred while each accident hotspot was encountered. In the following section, we discuss the regression results shown in Table 3.

Firstly, in Model (1) the regression was run with only the independent variable 'warning' capturing whether or not the warning intervention was provided to the driver, and thus the difference between the control and intervention groups. Here we do not see

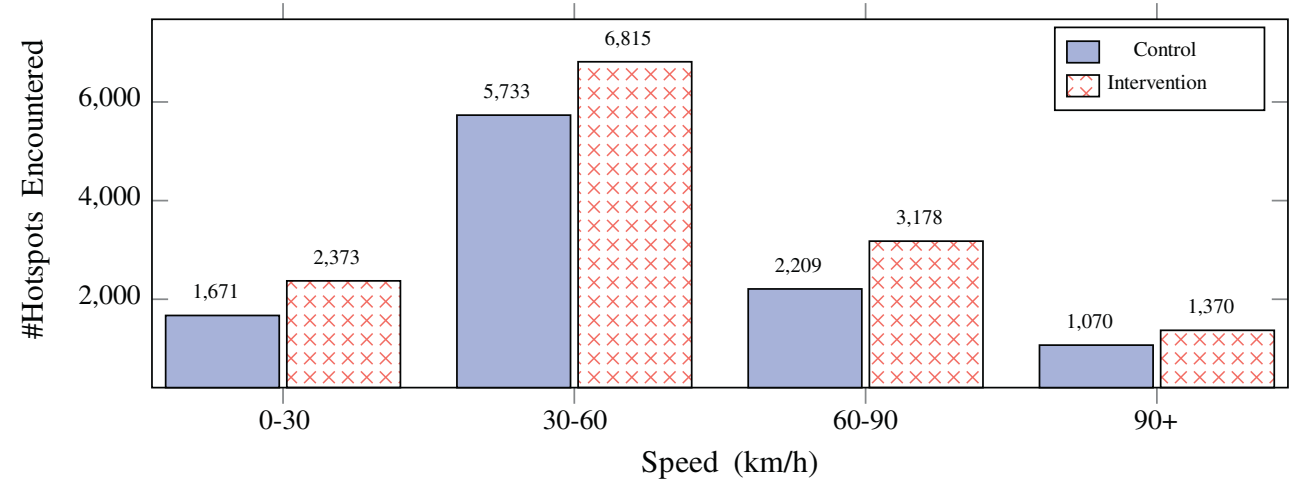

Fig. 4. Distribution of speed and number of hotspots for control and intervention groups. 
Table 3

Binary logistic regression odds ratio and significance. dependent variable: occurrence of a dangerous or high danger braking event. $\mathrm{N}=24,419$.

\begin{tabular}{|c|c|c|c|c|c|c|c|}
\hline & (1) & (2) & (3) & (4) & (5) & (6) & (7) \\
\hline Warning & 0.950 & 1.115 & 1.081 & 1.124 & 0.997 & 0.976 & 1.021 \\
\hline Number of warnings & & $0.892^{* * *}$ & $0.922^{* *}$ & $0.892^{* * *}$ & $0.891^{* * *}$ & $0.921^{* *}$ & 1.276 \\
\hline \multicolumn{8}{|l|}{ Speed (vs. 0-30km/h) } \\
\hline $30-60 \mathrm{~km} / \mathrm{h}$ & & & $2.008^{* * * *}$ & & & $2.035^{* * * *}$ & $2.031^{* * * *}$ \\
\hline $60-90 \mathrm{~km} / \mathrm{h}$ & & & $1.611^{* * * *}$ & & & $1.632^{* * * *}$ & $1.632^{* * * *}$ \\
\hline $90+\mathrm{km} / \mathrm{h}$ & & & $0.472^{* * *}$ & & & $0.477^{* * * *}$ & $0.479^{* * * *}$ \\
\hline \multicolumn{8}{|l|}{ Time of day (vs. 00-05h) } \\
\hline $05-18 \mathrm{~h}$ & & & & 1.038 & & 1.054 & 1.049 \\
\hline $18-21 \mathrm{~h}$ & & & & 0.874 & & 0.876 & 0.876 \\
\hline $21-24 \mathrm{~h}$ & & & & 0.768 & & 0.759 & 0.765 \\
\hline \multicolumn{8}{|l|}{ Day of week (vs. Monday) } \\
\hline Tuesday & & & & $1.183^{*}$ & & $1.212^{*}$ & $1.219^{*}$ \\
\hline Wednesday & & & & 1.078 & & 1.102 & 1.108 \\
\hline Thursday & & & & 1.067 & & 1.094 & 1.101 \\
\hline Friday & & & & 1.107 & & 1.126 & 1.128 \\
\hline Saturday & & & & 0.959 & & 0.970 & 0.976 \\
\hline Sunday & & & & 1.127 & & 1.143 & 1.148 \\
\hline \multicolumn{8}{|l|}{ Driver age (vs. 18-25) } \\
\hline $25-35$ & & & & & 0.781 & 0.718 & 0.734 \\
\hline $35-59$ & & & & & 0.803 & 0.717 & 0.714 \\
\hline $59+$ & & & & & 0.895 & 0.813 & 0.830 \\
\hline \multicolumn{8}{|l|}{ Driver personality } \\
\hline Agreeableness & & & & & $0.913^{*}$ & $0.913^{*}$ & 0.954 \\
\hline Conscientiousness & & & & & 1.038 & 1.034 & 1.023 \\
\hline Extraversion & & & & & 1.001 & 0.996 & 1.006 \\
\hline Neuroticism & & & & & 1.046 & 1.049 & 1.037 \\
\hline Openness & & & & & 0.974 & 0.986 & 0.985 \\
\hline \multicolumn{8}{|l|}{ Driver age interactions (vs. 18-25) } \\
\hline Number of warnings $\times 25-35$ & & & & & & & 0.930 \\
\hline Number of warnings $\times 35-59$ & & & & & & & 1.000 \\
\hline Number of warnings $\times 59+$ & & & & & & & 0.978 \\
\hline \multicolumn{8}{|l|}{ Driver personality interactions } \\
\hline Number of warnings $\times$ Agreeableness & & & & & & & $0.944^{*}$ \\
\hline Number of warnings $\times$ Conscientiousness & & & & & & & 1.013 \\
\hline Number of warnings $\times$ Extraversion & & & & & & & 0.988 \\
\hline Number of warnings $\times$ Neurotiscism & & & & & & & 1.005 \\
\hline Number of warnings $\times$ Openness & & & & & & & 0.994 \\
\hline Constant & $0.164^{* * *}$ & $0.164^{* * *}$ & $0.103^{* * * *}$ & $0.151^{* * *}$ & $0.266^{* *}$ & $0.158^{* * * *}$ & $0.128^{* * * *}$ \\
\hline L1 error & $0.354^{* * * *}$ & $0.350^{* * *}$ & $0.338^{* * *}$ & $0.353^{* * *}$ & $0.324^{* * *}$ & $0.314^{* * * *}$ & $0.317^{* * * *}$ \\
\hline
\end{tabular}

$* p<0.05$.

** $p<0.01$.

*** $p<0.001$.

any significant impact of the warnings provided. This indicates that when only comparing the braking behaviour between the control group and the intervention group, the occurrence of a warning had no significant impact on driver safety. Thus, we are unable to confirm the immediate positive effect of warnings as seen across many lab studies.

Exploring this further in Model (2), we add to the previous analysis an additional independent variable 'number of warnings'. This variable describes the number of times a driver in the intervention group has been shown the warning for a specific accident hotspot. This way we can explore the learning effect that repeated warnings of the same area have on a driver. Instead of linear effects of the number of warnings experienced, we expect the effectiveness of repeated warnings to decrease with each additional warning experienced. Thus, in line with existing studies $[39,40]$, we examine the time effects of the variable using a logarithmic transformation. Here we see that the number of times a warning has been shown has a significant effect on dangerous braking behaviour. This indicates that the more times a driver is exposed to the same warning in a hazardous area, the more cautious he drives and the less likely he is to have a dangerous braking event. Overall this is a positive result, and shows that the accident hotspot warning intervention has a significant learning effect on driving behaviour over time, but not an immediate short-term one.

Various models were analysed to test whether the significance of the learning effect of the in-vehicle warnings remained stable. As additional independent variables we also considered the speed that the vehicle was travelling when the warning was shown, the time of day, day of week, driver age and each of the Big Five personality traits of the driver. Incorporating the speed as a predictor into Model (3), we see that the immediate warning effect continues to be insignificant, and the learning effect remains. Additionally we observe significant variations in the likelihood of a heavy braking event based on the speed that an accident hotspot was approached at. The stability of the learning effect was also tested with temporal variables in Model (4), which have historically been found to influence the rate of crashes [39]. Both time of day and day of week categorical independent variables were incorporated into the regression model. The short-term warning continues to be insignificant, and the learning effect remains at the same level as seen in Model 
(2). Overall, in contrast to previous studies, no time of day category was more or less dangerous at a significant level when compared to the '00-05 h' category. The only significant temporal effect was comparing the days of the week, Monday compared to Tuesday, where on Tuesday it was found to be more likely to encounter a dangerous braking event.

Various studies have shown the effect of a driver's age and personality factors on driving related behaviour [55]. In order to incorporate this into our model, we added age categories and personality information of the drivers in Model (5). As with the other models, the short-term effect of the warnings remained insignificant and the learning effect remained significant at a similar level to Models (2) and (4). Although investigating the effect of driver personality on driving behaviour is not the primary aim of this paper, based on our sample of drivers we are able to see a significant effect of Agreeableness reducing the likelihood of a dangerous braking event. This seems to confirm findings from a previous study [55], where low levels of Agreeableness are correlated to 'reckless' and 'angry' driving styles, and high levels correlate to 'careful' driving behaviour.

Each of the additional independent variables discussed in Models (3), (4) and (5) were merged into the combined Model (6). We continue to see the insignificant immediate effect of the intervention and the learning effect remains stable across all the models, showing a reduction in the likelihood of a dangerous braking event. The other significant effects discussed in the previous models also remain in the fully merged model, and no new features become significant.

Existing evidence suggest that an individual's characteristics, such as age and personality, are important variables which might affect the generalizability of our findings, for example, in the form of moderators [52,55,58]. Thus, we enhance Model (6) with interactions between the observed learning effect and age, as well as personality. Specifically, the interactions between the "number of warnings' and driver age and personality variables were added to generate Model (7). Here we see that neither the learning effect nor the effect of Agreeableness remain significant. However, the interaction between the two is. This interaction indicates that the learning effect we have identified is dependent on an individual's level of Agreeableness, where only those with reasonable levels improve their driving behaviour due to the warnings provided by the system.

\section{Discussion, limitations and future work}

Summing up, we demonstrated in a large field study that in-vehicle warnings of accident hotspots can have a significant improvement on driver behaviour over time. However, we did not see an immediate positive or negative effect of the warnings on dangerous braking behaviour of the drivers. When investigating generalizability on the basis of interactions, results indicate that the learning effect requires an adequate level of driver Agreeableness. The Agreeableness personality trait is linked to characteristics such as cooperation and social harmony [20]. Hence, drivers who do not accept advice from an in-vehicle DSS (lack of willingness to cooperate) or do not care or reflect that they might harm others (lack of social harmony) might not benefit from such a system. Thus, in order to improve driver safety, future work should investigate the key determinants of, and how to best facilitate, the learning effects of such in-vehicle warning DSSs [5,6]. Finally, although the impact of vehicle speed on the likelihood of heavy braking events is not a focus of this paper, we find a significant effect in our data, which is similar to previous work that considers the influence of velocity on the exposure-accident relationship [39]. In line with our findings, the literature generally associates higher velocities with a greater risk of accident involvement [1]. This is primarily due to larger stopping distances and reduced manoeuvrability at higher speeds.
The presented work has implications both for researchers and practitioners. From a research perspective, the learning effect we observe is well known with regard to digital interventions. Similar long-term effects are seen in other domains, such as health [6] and education [5], where significant effects are reported the more often an intervention was triggered. Our results further confirm the importance of measuring personality traits when researching interventions with DSSs. Personality traits have long been recognized as a strong predictor of human decision-making outcomes [52]. However, research on real-time feedback interventions have only recently considered the impact of personality as a key factor in human behaviour [58]. Additionally, the results emphasize the importance of field research. The large effects that are often reported from very controlled settings have to be verified under real-world conditions to ensure generalizability. For practitioners, the main implication of our results is that accident hotspot warnings can improve driver and traffic safety over time. Policy makers should seriously consider promoting in-vehicle DSSs, since such systems are lightweight, low cost and highly scalable [6]. Hence, they could efficiently complement traditionally more complex and expensive approaches to improve traffic safety, such as changing hazardous road infrastructure. For vehicle manufacturers and mobility solution providers the presented accident hotspot warning could extend their portfolio of safety features and connected car services, both of which are increasingly important for car buyers' purchase decisions [62]. On a more general note, the interaction of Agreeableness and the learning effect that we see in the results imply a call for action towards the personalization of DSSs, as the effectiveness of DSS-based interventions vary according to an individual's characteristics [58]. However, measuring personality traits is inconvenient and often perceived as intrusive by the user [59]. As such, one can either seek to identify the user's personality unobtrusively, or rely on the stable effect of the warnings over time and on the consumers' self-selection.

The results of this study should be seen in the light of its limitations. The system makes use of historical accident data from a national dataset, limiting the adaptation of this approach to regions with similar sources of information. However, there is increasing work in identifying accident hotspots from near-miss events detected through connected vehicles, thus removing the need for historical data. Another key weakness is the homogeneity of the researched sample. The participants who evaluated the system were all male and professional drivers. Their profession means that they are overall expected to be more experienced and safer drivers, thus this sample may not be easily generalizable to regular drivers. The proficiency of our sample of drivers, however, implies that we are likely to have underestimated the effect of our solution, as there is more potential to improve driving behaviour for more regular, less proficient drivers. Furthermore, this research is geared towards the development and validation of an innovative artefact. In accordance with this goal and in conformance with latest discussions in the scientific community [61], the paper does not focus on theory. Future research should cover theoretical models of human behaviour to further increase generalizability of the findings. Finally, the sample size of the study was fairly small, further studies should make use of a larger and more diverse sample of drivers for more reliable and generalizable results.

\section{Conclusions}

In-vehicle DSSs can encourage drivers to adapt their driving behaviour when necessary, and have therefore been the focus of various research endeavours. Latest studies provide promising evidence that these systems can indeed have significant positive effects on driving behaviour and collision avoidance. Going beyond existing research, a complete in-vehicle DSS was designed and implemented, which provides accident hotspot warnings to drivers based 
on location analytics applied to a national historical accident dataset. The system was tested with 57 drivers in a field test covering over $170,000 \mathrm{~km}$. As such, the paper at hand is among the first to bring research on in-vehicle DSSs and warnings for drivers into the field in a realistic experimental setting.

Ultimately, we find that in-vehicle warnings of accident hotspots have a significant improvement on driver behaviour over time. Thereby, we demonstrate that DSSs and design research can play a fruitful role in the field of connected vehicles, a domain which has traditionally not been a core focus of DSSs and information systems research. In addition, we find that positive intervention effects are bound to drivers' Agreeableness, i.e. drivers have to be willing to "listen" to the in-vehicle DSS. Hence, future research should carefully reflect the role and impact of subjects' Agreeableness. Moreover, we see a potential for design science research to develop and validate effective strategies that help to overcome technology adoption challenges, which are based on a lack of Agreeableness.

This research is highly relevant to both policy makers and industry players, such as vehicle manufactures and insurances. Numerous hardware-based vehicle safety systems have become mandatory in various countries throughout the last decades, for example, air-bags and electronic stability programs. Similarly, policy makers should now consider promoting data-driven in-vehicle DSSs. Eventually, in-vehicle DSSs that have proven to prevent accidents could also be enforced by corresponding regulation. The automotive industry should recognize that data-driven prevention services might be an effective means to address the distinct safety needs of consumers and form a basis for sustainable competitive differentiation.

Finally, in contrast to existing lab experiments with very promising results, we were not able to confirm an immediate effect of warnings on driver behaviour. This demonstrates the importance of building innovative artefacts and conducting experimental research in a realistic field setting. Thus, we see a strong need for further field experiments with high resolution car data to confirm that in-vehicle DSSs can deliver in diverse field situations.

\section{Acknowledgments}

The authors would like to acknowledge the feedback, comments and suggestions from two anonymous reviewers and the co-editor of the special issue specifically, whose input has greatly improved this paper. This work is part of the Bosch IoT Lab, headed by Prof. Dr. Elgar Fleisch at the University of St. Gallen, Switzerland. We would also like to thank our colleagues on our Connected Car project, Prof. Dr. Markus Weinberger and Timo Gessmann.

\section{References}

[1] L. Aarts, I. Van Schagen, Driving speed and the risk of road crashes: a review, Accid. Anal. Prev. 38 (2006) 215-224.

[2] P.E. An, C.J. Harris, An intelligent driver warning system for vehicle collision avoidance, IEEE Trans. Syst. Man Cybern. Part A Syst. Hum. 26 (1996) 254-261.

[3] T.K. Anderson, Kernel density estimation and K-means clustering to profile road accident hotspots, Accid. Anal. Prev. 41 (2009) 359-364.

[4] L.M. Bergasa, D. Almería, J. Almazán, J.J. Yebes, R. Arroyo, Drivesafe: an app for alerting inattentive drivers and scoring driving behaviors, 2014 IEEE Intelligen Vehicles Symposium Proceedings, IEEE. 2014, pp. 240-245.

[5] C. Bokhove, P. Drijvers, Effects of a digital intervention on the development of algebraic expertise, Comput. Educ. 58 (2012) 197-208.

[6] H. Brendryen, P. Kraft, Happy ending: a randomized controlled trial of a digita multi-media smoking cessation intervention, Addiction 103 (2008) 478-484.

[7] J.L. Campbell, C.M. Richard, J.L. Brown, M. McCallum, Crash warning system interfaces: human factors insights and lessons learned, NHTSA report\# HS 810 (2007) 697.

[8] J. Coutinho-Rodrigues, J. Current, J. Climaco, S. Ratick, Interactive spatial decision-support system for multiobjective hazardous materials location-routing problems, Transp. Res. Rec. J. Transp. Res. Board (1997) 101-109.
[9] J.A. Deacon, C.V. Zegeer, R.C. Deen, Identification of Hazardous Rural Highway Locations, Transportation Research Board. 1974.

[10] M. Deublein, M. Schubert, B.T. Adey, B.G. de Soto, A Bayesian network model to predict accidents on Swiss highways, Infrastructure Asset Manage. 2 (2015) $145-158$.

[11] S. Erdogan, I. Yilmaz, T. Baybura, M. Gullu, Geographical information systems aided traffic accident analysis system case study: city of Afyonkarahisar, Accid. Anal. Prev. 40 (2008) 174-181.

[12] M. Ester, H.-P. Kriegel, J. Sander, X. Xu, et al. A Density-Based Algorithm for Discovering Clusters in Large Spatial Databases with Noise., Kdd, 96, 1996. pp. 226-231.

[13] B. Flahaut, M. Mouchart, E. San Martin, I. Thomas, The local spatial autocorrelation and the kernel method for identifying black zones: a comparative approach, Accid. Anal. Prev. 35 (2003) 991-1004.

[14] K. Geurts, I. Thomas, G. Wets, Understanding spatial concentrations of road accidents using frequent item sets, Accid. Anal. Prev. 37 (2005) 787-799.

[15] E. Hauer, Observational Before/After Studies in Road Safety. Estimating the Effect of Highway and Traffic Engineering Measures on Road Safety, Elsevier. 1997.

[16] E. Hauer, D. Harwood, F. Council, M. Griffith, Estimating safety by the empirical Bayes method: a tutorial, Transp. Res. Rec. J. Transp. Res. Board (2002) 126131.

[17] E. Hauer, B.N. Persaud, Problem of Identifying Hazardous Locations Using Accident Data, Transportation Research Board. 1984.

[18] S. Hirst, R. Graham, The Format and Presentation of Collision Warnings, Ergonomics and safety of intelligent driver interfaces, 1997, 203-219.

[19] T. Imkamon, P. Saensom, P. Tangamchit, P. Pongpaibool, Detection of hazardous driving behavior using fuzzy logic, Electrical Engineering/Electronics, Computer, Telecommunications and Information Technology, 2008. ECTI-CON 2008. 5Th International Conference On, 2, IEEE. 2008, pp. 657-660.

[20] O.P. John, S. Srivastava, The big five trait taxonomy: history, measurement, and theoretical perspectives, Handb. Pers. Theory Res. 2 (1999) 102-138.

[21] D.A. Johnson, M.M. Trivedi, Driving style recognition using a smartphone as a sensor platform, 2011 14Th International IEEE Conference on Intelligent Transportation Systems (ITSC), IEEE. 2011, pp. 1609-1615.

[22] O. Karaduman, H. Eren, H. Kurum, M. Celenk, An effective variable selection algorithm for aggressive/calm driving detection via CAN Bus, 2013 International Conference on Connected Vehicles and Expo (ICCVE), IEEE. 2013, pp. 586-591.

[23] J. Kazazi, S. Winkler, M. Vollrath, Accident prevention through visual warnings: how to design warnings in head-up display for older and younger drivers, 2015 IEEE 18Th International Conference on Intelligent Transportation Systems, IEEE. 2015, pp. 1028-1034.

[24] J. Kazazi, S. Winkler, M. Vollrath, Accident prevention through visual warnings: how to design warnings in head-up display for older and younger drivers, 2015 IEEE 18Th International Conference on Intelligent Transportation Systems, 2015. pp. 1028-1034.

[25] P.B. Keenan, Spatial decision support systems for vehicle routing, Decis. Support. Syst. 22 (1998) $65-71$.

[26] K. Khan, S.U. Rehman, K. Aziz, S. Fong, S. Sarasvady, DBSCAN: past, present and future, Applications of Digital Information and Web Technologies (ICADIWT), 2014 Fifth International Conference on The, IEEE. 2014, pp. 232-238.

[27] L. Lam, S. Suen, Application of majority voting to pattern recognition: an analysis of its behavior and performance, IEEE Trans. Syst. Man Cybern. Part A Syst. Humans 27 (1997) 553-568

[28] K. McMahon, S. Dahdah, The True Cost of Road Crashes: Valuing Life and the Cost of a Serious Injury, iRAP, London, 2008.

[29] F. Meng, C. Spence, Tactile warning signals for in-vehicle systems, Accid. Anal. Prev. 75 (2015) 333-346.

[30] S.-P. Miaou, The relationship between truck accidents and geometric design of road sections: Poisson versus negative binomial regressions, Accid. Anal. Prev. 26 (1994) 471-482.

[31] A. Montella, A comparative analysis of hotspot identification methods, Accid. Anal. Prev. 42 (2010) 571-581.

[32] F. Naujoks, H. Grattenthaler, A. Neukum, G. Weidl, D. Petrich, Effectiveness of advisory warnings based on cooperative perception, IET Intell. Transp. Syst. 9 (2015) 606-617.

[33] F. Naujoks, A. Neukum, Specificity and timing of advisory warnings based on cooperative perception., Mensch \& Computer Workshopband, 2014. pp. 229-238.

[34] F. Naujoks, A. Neukum, Timing of in-vehicle advisory warnings based on cooperative perception, Proceedings of the Human Factors and Ergonomics Society Europe Chapter Annual Meeting, 2014.

[35] F. Naujoks, I. Totzke, Behavioral adaptation caused by predictive warning systems-the case of congestion tail warnings, Transport. Res. F: Traffic Psychol. Behav. 26 (2014) 49-61.

[36] NHTSA, 2015 Motor vehicle crashes: Overview (2015), 2015, https://crashstats. nhtsa.dot.gov/Api/Public/ViewPublication/812318. accessed 16.09.2016.

[37] A. Okabe, T. Satoh, K. Sugihara, A kernel density estimation method for networks, its computational method and a GIS-based tool, Int. J. Geogr. Inf. Sci. 23 (2009) 7-32.

[38] S. Oppe, The use of multiplicative models for analysis of road safety data, Accid. Anal. Prev. 11 (1979) 101-115.

[39] J. Paefgen, T. Staake, E. Fleisch, Multivariate exposure modeling of accident risk: insights from pay-as-you-drive insurance data, Transp. Res. A Policy Pract. 61 (2014) 27-40. 
[40] J. Paefgen, T. Staake, F. Thiesse, Evaluation and aggregation of pay-as-youdrive insurance rate factors: a classification analysis approach, Decis. Support. Syst. 56 (2013) 192-201.

[41] V. Prasannakumar, H. Vijith, R. Charutha, N. Geetha, Spatio-temporal clustering of road accidents: GIS based analysis and assessment, Procedia - Social and Behavioral Sciences, 21, 2011. pp. 317-325.

[42] S.S. Pulugurtha, V.K. Krishnakumar, S.S. Nambisan, New methods to identify and rank high pedestrian crash zones: an illustration, Accid. Anal. Prev. 39 (2007) 800-811.

[43] B. Rammstedt, O.P. John, Measuring personality in one minute or less: a 10-item short version of the big five inventory in english and german, J. Res. Pers. 41 (2007) 203-212.

[44] J.J. Ray, A web-based spatial decision support system optimizes routes for oversize/overweight vehicles in Delaware, Decis. Support. Syst. 43 (2007) 1171-1185.

[45] D. Ruscio, M.R. Ciceri, F. Biassoni, How does a collision warning system shape driver's brake response time? The influence of expectancy and automation complacency on real-life emergency braking, Accid. Anal. Prev. 77 (2015) 72-81.

[46] B. Ryder, B. Gahr, A. Dahlinger, An in-vehicle information system providing accident hotspot warnings, Twenty-Fourth European Conference on Information Systems (ECIS), AIS. 2016

[47] B. Ryder, B. Gahr, P. Egolf, A. Dahlinger, F. Wortmann, Preventing traffic accidents with in-vehicle decision support systems - warning sign and text combinations, 2017, http://www.iot-lab.ch/wp-content/uploads/2017/02/warning signs_and_text.pdf. accessed 15.02.2017.

[48] L. Santos, J. Coutinho-Rodrigues, C.H. Antunes, A web spatial decision support system for vehicle routing using Google maps, Decis. Support. Syst. 51 (2011) $1-9$.

[49] F. Seeliger, G. Weidl, D. Petrich, F. Naujoks, G. Breuel, A. Neukum, K. Dietmayer, Advisory warnings based on cooperative perception, Intelligent Vehicles Symposium, 2014. pp. 246-252.

[50] V. Shankar, F. Mannering, W. Barfield, Effect of roadway geometrics and environmental factors on rural freeway accident frequencies, Accid. Anal. Prev. 27 (1995) 371-389.

[51] T.A. Snijders, Multilevel Analysis, Springer. 2011.

[52] G.B. Sprotles, E.L. Kendall, A methodology for profiling consumers' decisionmaking styles, J. Consum. Aff. 20 (1986) 267-279.

[53] S. Szénási, P. Csiba, Clustering algorithm in order to find accident black spots identified by gps coordinates, 14th SGEM GeoConference on Informatics, Geoinformatics and Remote Sensing, 1, 2014. pp. 505-512.

[54] S. Szénási, D. Jankó, A method to identify black spot candidates in built-up areas, Journal of Transportation Safety \& Security (2016) 1-25.

[55] O. Taubman-Ben-Ari, D. Yehiel, Driving styles and their associations with personality and motivation, Accid. Anal. Prev. 45 (2012) 416-422.

[56] L.S. Tey, L. Ferreira, A. Wallace, Measuring driver responses at railway level crossings, Accid.Anal. Prev. 43 (2011) 2134-2141.

[57] L.S. Tey, S. Zhu, L. Ferreira, G. Wallis, Microsimulation modelling of driver behaviour towards alternative warning devices at railway level crossings, Accid. Anal. Prev. 71 (2014) 177-182.

[58] V. Tiefenbeck, L. Goette, K. Degen, V. Tasic, E. Fleisch, R. Lalive, T. Staake, Overcoming salience bias: how real-time feedback fosters resource conservation, Manag. Sci. (2016) (mnsc.2016.2646).

[59] R. Tourangeau, T. Yan, Sensitive questions in surveys., Psychol. Bull. 133 (2007) 859.

[60] F. Ülengin, Ș. Önsel, Y.I. Topçu, E. Aktaş, Ö. Kabak, An integrated transportation decision support system for transportation policy decisions: the case of Turkey, Transp. Res. A Policy Pract. 41 (2007) 80-97.
[61] R.H. Von Alan, S.T. March, J. Park, S. Ram, Design science in information systems research, MIS Q. 28 (2004) 75-105.

[62] D. Wee, M. Kässer, M. Bertoncello, K. Heineke, G. Eckhard, J. Hölz, F. Saupe, T. Müller, Competing for the Connected Customer Perspectives on the Opportunities Created by Car Connectivity and Automation, McKinsey \& Company. 2015.

[63] J. Werneke, M. Vollrath, How to present collision warnings at intersections? a comparison of different approaches, Accid. Anal. Prev. 52 (2013) 91-99.

[64] J. Whitelegg, A geography of road traffic accidents, Trans. Inst. Br. Geogr. (1987) 161-176.

[65] WHO, Global status report on road safety, 2015, http://www.who.int/violence injury_prevention/road_safety_status/2015/en. accessed 24.02.2016.

[66] Z. Xie, J. Yan, Kernel density estimation of traffic accidents in a network space, Comput. Environ. Urban. Syst. 32 (2008) 396-406.

[67] H. Yu, P. Liu, J. Chen, H. Wang, Comparative analysis of the spatial analysis methods for hotspot identification, Accid. Anal. Prev. 66 (2014) 80-88.

[68] J. Zhang, K. Suto, A. Fujiwara, Effects of in-vehicle warning information on drivers' decelerating and accelerating behaviors near an arch-shaped intersection, Accid. Anal. Prev. 41 (2009) 948-958.

Benjamin Ryder is a doctoral student at Swiss Federal Institute of Technology (ETH) Zurich, Switzerland with the Chair of Information Management. He holds Bachelor's and Master's degrees in Computer Science from Imperial College London, where he graduated in 2012. His research interests are in data mining and statistical analysis, with a focus on vehicular and smartphone sensor data from which accident hotspots and driver profiles can be obtained. He is associated with the Bosch IoT Lab at ETH Zurich and the University of St. Gallen.

Bernhard Gahr is a doctoral student at the University of St. Gallen, Switzerland with the Chair of Operations Management. He holds Bachelor's and Master's degrees in engineering from the Swiss Federal Institute of Technology (ETH) Zurich, where he graduated in 2015. His research interests are in the potential of the connected car for improving self-driving technology and safe driving performance. He is associated with the Bosch IoT Lab at ETH Zurich and the University of St. Gallen.

Philipp Egolf is a student at the Swiss Federal Institute of Technology (ETH) Zurich, Switzerland with the Chair of Information Management. He holds Bachelor's degree in Mechanical Engineering from ETH Zurich. His research interests are in spatial data mining and smartphone sensor analytics, from which hazardous locations and driving events can be obtained.

Andre Dahlinger is a doctoral student at the University of St. Gallen, Switzerland with the Chair of Operations Management. He holds a Diploma (M.Sc.) in psychology and a B.Sc. in economics from the Philipps-University of Marburg. His research interests are in the potential of the connected car for improving driving performance, ecodriving and car security. He is associated with the Bosch IoT Lab at ETH Zurich and the University of St. Gallen.

Felix Wortmann is assistant professor at the Institute of Technology Management University of St. Gallen (HSG) and the scientific director of the Bosch Internet of Things Lab. His research interests include Big Data and the Internet of Things. Before that he worked as an assistant to the executive board of SAP. Felix Wortmann received his Bachelor's and Master's degree in Information Systems from the University of Muenster, Germany, and a PhD in Management from the University of St. Gallen. 